\title{
Segmental orientation of a single polymer chain in Langmuir-Blodgett films studied by defocus fluorescence imaging
}

Polymer Journal (2011) 43, 218-221; doi:10.1038/pj.2010.125; published online 8 December 2010

\section{INTODUCTION}

Amphiphilic polymers, such as poly(alkyl methacrylate)s and poly(alkyl acrylamide)s, are known to form stable monolayers on a water surface by the Langmuir-Blodgett (LB) method. ${ }^{1,2}$ The polymer monolayer has an ultimate thinness of the size of the monomer unit, and it has few defects in the film plane. Therefore, the monolayer of an amphiphilic polymer has attracted much interest as an ideal building block to fabricate well-designed layered structures at a nanometric scale. In a monolayer of a polymer consisting of amphiphilic monomers such as alkyl ester and amide groups, each monomer unit is strongly adsorbed onto a water surface. Consequently, the main chain is restricted in a two-dimensional plane, resulting in a greatly reduced degree of freedom compared with a threedimensional bulk state. The macroscopic properties of the polymeric materials are closely related to the conformation of the polymer chain; the polymer monolayer shows different properties from the threedimensional bulk system. ${ }^{3-8}$ Therefore, it is important to understand the conformation and orientation of the polymer main chain in a monolayer at the single molecule level. The chain structures in LB films have been extensively studied by various methods such as infrared and optical spectroscopy. ${ }^{9-12}$ The polarization dichroism of the absorption spectra provides information on the average orientation of functional groups in the polymer chain. However, because of the weak signal intensity from the ultrathin films, the observation has been limited to the chemical moieties with a strong absorption coefficient, such as a carbonyl group in infrared spectroscopy.

The fluorescence labeling technique is useful for probing the local structure of polymer monolayers because of the high sensitivity in detecting fluorescence emissions. ${ }^{13}$ In addition to high sensitivity, the appropriate molecular design to introduce the fluorescent moiety allows the selective measurement of a specific site in the system. Highly sensitive optical imaging devices have enabled the direct observation of a fluorescent molecule; therefore, fluorescence microscopy would be a powerful tool to study the chain structure in an LB film at the single chain level. In a conventional fluorescence image, each molecule is observed as a circular spot. However, slight deviation of the objective of the microscope from the focal condition can change the observed pattern of a single molecule, which is strongly dependent on the orientation of the transition dipole. ${ }^{14}$ Consequently, the image analysis of the defocus pattern provides information on the orientation of a single dye molecule. This technique determines both the polar and azimuthal angles $(\theta, \varphi)$ of an individual molecule from the fluorescence image. Such defocus imaging techniques have been used to discuss the molecular orientation of dye molecules doped in thin films and molecular motor systems. ${ }^{15-19}$

The current study focused on the orientation of the polymer main chain in an LB film. The conformation of the main chain is closely related to its macroscopic physical properties; however, the direct evaluation of the orientation of the backbone segment has been difficult. We previously reported the synthesis of the poly(methacrylate)s labeled by a perylene diimide (PDI) molecule at the center of the chain, where the transition dipole of PDI was incorporated into the backbone of the polymer chain. ${ }^{15}$ The defocus imaging of the center-labeled polymer chain enabled the discussion of the segmental orientation of a single polymer chain. In this paper, the direct evaluation of the orientation of the single backbone segment of the poly(methyl methacrylate) (PMMA) chain in an LB film was demonstrated. The molecular orientation of the center segment of the PMMA chain was quantitatively evaluated by the defocus fluorescence imaging technique, and the effect of the deposition method is discussed.

\section{EXPERIMENTAL PROCEDURE}

The PMMA chain labeled with a single PDI molecule (Figure 1) was prepared by atom transfer radical polymerization from the difunctional PDI initiator, which was synthesized by the imidization of 3,4,9,10-perylenetetracarboxlic dianhydride with $(S)-(+)$ leucinol, followed by the reaction with 2bromopropyonyl bromide. The polymerization of methyl methacrylate with $\mathrm{CuCl}(\mathrm{I})$ / dinonylbipyridyl yielded a fluorescencelabeled PMMA chain at the center of the main chain. ${ }^{15}$ The weight- and number-average molecular weights were evaluated to be $1.23 \times 10^{5}$ and $9.8 \times 10^{4}$, respectively, by sizeexclusion chromatography calibrated with PMMA standards (Scientific Polymer Product, Ontario, NY, USA). The labeled chain was mixed with unlabeled PMMA with an $M_{\mathrm{w}}$ of $10^{5}$ at a ratio of $0.05 \%$. A benzene solution of the polymer mixture at a concentration of $0.1 \mathrm{gl}^{-1}$ was spread on pure water to form a monolayer. The surface pressurearea isotherm is shown in Supplementary Information. After compression up to a surface pressure of $5 \mathrm{mN} \mathrm{m}^{-1}$, the monolayer was transferred onto a glass coverslip by vertical and horizontal dipping methods, which were denoted as $\mathrm{V}$ - and $\mathrm{H}$-films, respectively. In vertical dipping, the substrate was immersed vertically in the water 
subphase before spreading the PMMA solution. The monolayer was transferred by lifting the substrate out of the subphase. In the horizontal process, the monolayer was deposited by touching the substrate plane to the monolayer in parallel. Before lifting off the substate, the residual monolayer was removed from the water surface.

Fluorescence imaging was carried out by an inverted fluorescence microscope (TE-2000,

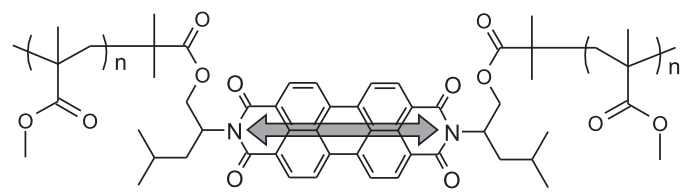

Figure 1 Chemical structure of the perylene diimide (PDI)-labeled poly(methyl methacrylate) at the chain center. The double-headed arrow indicates the transition dipole moment of the PDI moiety.
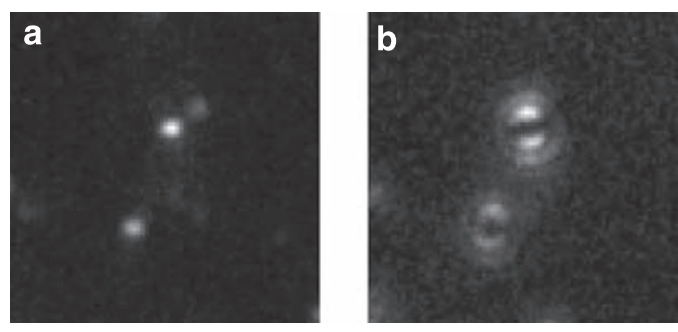

\section{C}

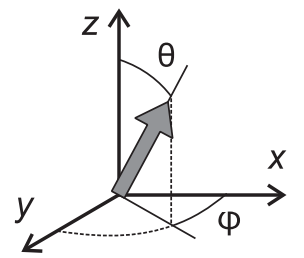

Figure 2 Fluorescence images of perylene diimide molecules in in-focus (a) and out-of-focus (b) conditions. The defocus image was obtained with the objective shift of $900 \mathrm{~nm}$ toward the sample. The polar and azimuthal angles $\theta$ and $\varphi$ defined in the (c) are evaluated to be $\left(96^{\circ}, 101^{\circ}\right)$ and $\left(97^{\circ}, 77^{\circ}\right)$ for the upper and lower molecules shown in the (a), respectively.
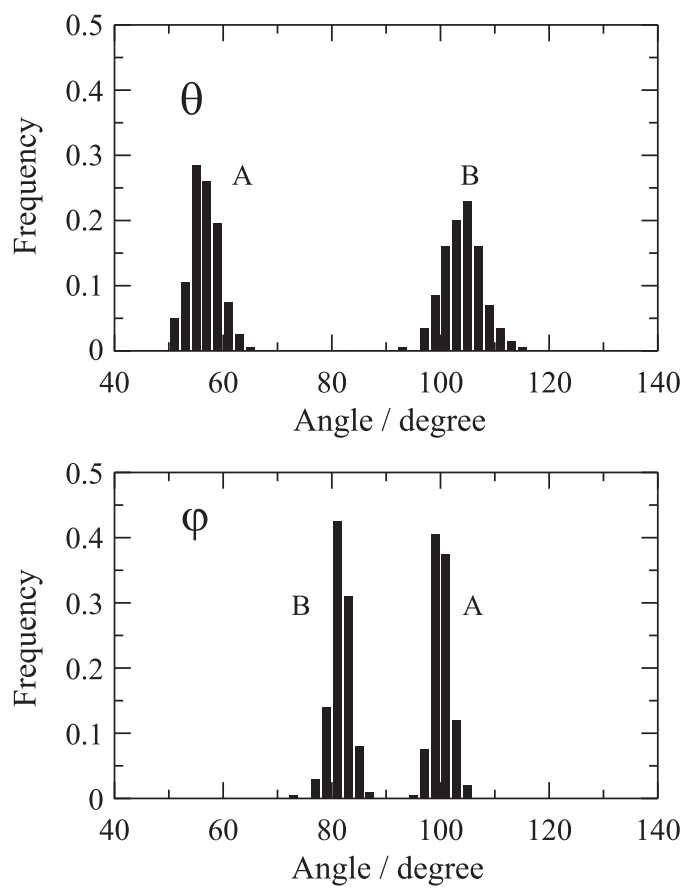

Figure 3 Histogram of the molecular orientation for two perylene diimide molecules A and B. The width for each histogram indicates the uncertainty of the apparatus.
Nikon, Tokyo, Japan). A circularly polarized $532-\mathrm{nm}$ laser beam at a power of $10 \mathrm{~mW}$ (GSHG-3015, Kochi Toyonaka Giken, Kochi, Japan) incident on the sample in a total internal reflection condition in an objective lens $(\times 100,1.4 \mathrm{NA}$, Nikon) was used to illuminate an area of $\sim 400 \mu \mathrm{m}^{2}$. The objective was mounted on a piezo actuator (P-721, Physik Instrumente, Karlsruhe, Germany), which enabled precise focus control with a resolution of $10 \mathrm{~nm}$. The fluorescence image was acquired by an EMCCD camera (Cascade II, Photometrics, Tucson, AZ, USA) through a dichroic mirror, a band-pass filter (z532rdc and HQ580/60 m, Chroma Technology, Brattleboro, VT, USA) and a $\times 2.5$ magnifier lens. Image analysis was carried out by homemade software written in Delphi and Scilab.

\section{RESULTS AND DISCUSSION}

Figure 2 shows the fluorescence images of the PDI units introduced at the center segment of the PMMA chains. In the image acquired in the in-focus condition of Figure 2a, each molecule was observed to be of a circular shape with a diffraction-limited size. The number density of the observed molecule was $0.025-0.030$ chains per $\mu \mathrm{m}^{2}$. This is in good agreement with the density of the labeled chain calculated from the condition of the sample preparation, indicating that individual PMMA chains were imaged in the fluorescence micrograph. On shifting the objective of the microscope from the focal position, the fluorescence image became blurred; however, the defocus patterns for the single molecules were different from each other as shown in Figure 2b. The orientational angle of the transition dipole relative to the optical axis of the apparatus resulted in the emission pattern seen in Figure $2 \mathrm{~b}$. The defocus pattern of a single emitter can be calculated theoretically as a function of the molecular orientation and the distance of the objective shift; ${ }^{14}$ therefore, the three-dimensional orientation of the PDI moiety can be evaluated by fitting the experimental data to the calculated image with variable parameters of $\theta$ and $\varphi$. In the current system, the polar and azimuthal angles were defined in the ranges of $0^{\circ} \leqslant \theta<180^{\circ}$ and $0 \leqslant \varphi<180^{\circ}$ because the transition moment of fluorescence emission is a bi-directional vector. For example, the upper and lower molecules seen in Figure 2 were evaluated to have the orientation $(\theta, \varphi)$ of $\left(96^{\circ}, 101^{\circ}\right)$ and $\left(97^{\circ}, 77^{\circ}\right)$, respectively.

We will first discuss the accuracy of the orientational evaluation by this method. As a reference sample, a spin-cast film of PMMA containing a small amount of labeled chains 
was prepared. For a single PDI molecule, 200 images were sequentially acquired for $20 \mathrm{~s}$ with a time interval of $100 \mathrm{~ms}$ at room temperature. Because the measurement temperature was much lower than the glass transition temperature of PMMA (above $100{ }^{\circ} \mathrm{C}$ ), it is unlikely that the motion of the main chain of PMMA will be observed in the measurement time. Therefore, the deviation of the fitting result of the sequential images for the single molecule was regarded as the measure of experimental accuracy. Figure 3 shows the histograms of the fitting result for two typical molecules. The s.d.s $\sigma_{\theta}$ and $\sigma_{\varphi}$ for the polar and azimuthal angles, respectively, indicate the accuracy of the evaluation of the molecular orientation from a defocus fluorescence image: $\sigma_{\theta}=2.7^{\circ}$ and $\sigma_{\varphi}=1.8^{\circ}$ for molecule $\mathrm{A} ; \sigma_{\theta}=3.6^{\circ}$ and $\sigma_{\varphi}=1.9^{\circ}$ for $\mathrm{B}$. Average $\sigma$ was $2.9^{\circ}$ and $1.6^{\circ}$ for polar and the azimuthal angles, respectively. This indicates that the molecular orientation of a single fluorophore can be determined with the angular resolution of a few degrees by the current method.

As shown in Figure 1, the PDI moiety is introduced in the main chain of PMMA and the transition moment of the PDI moiety is

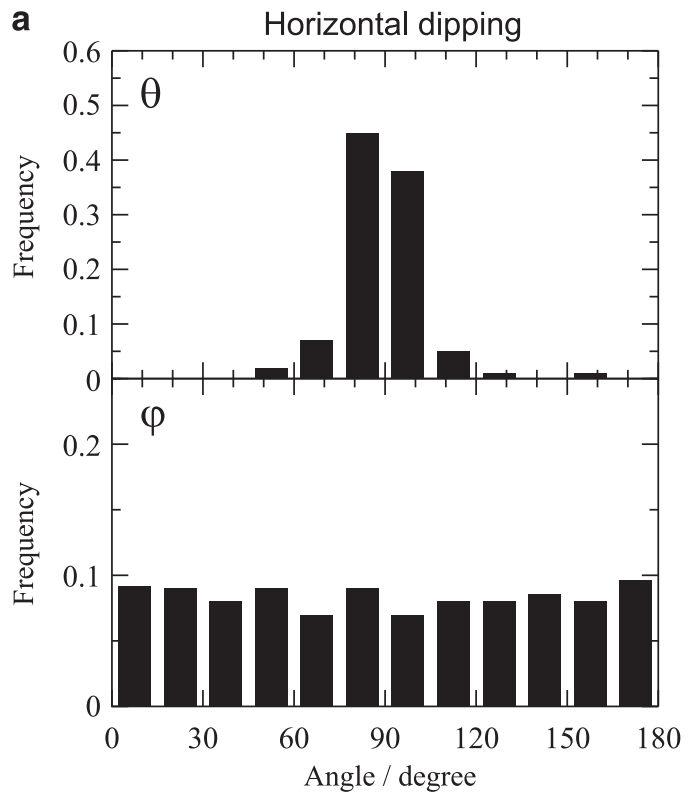

b

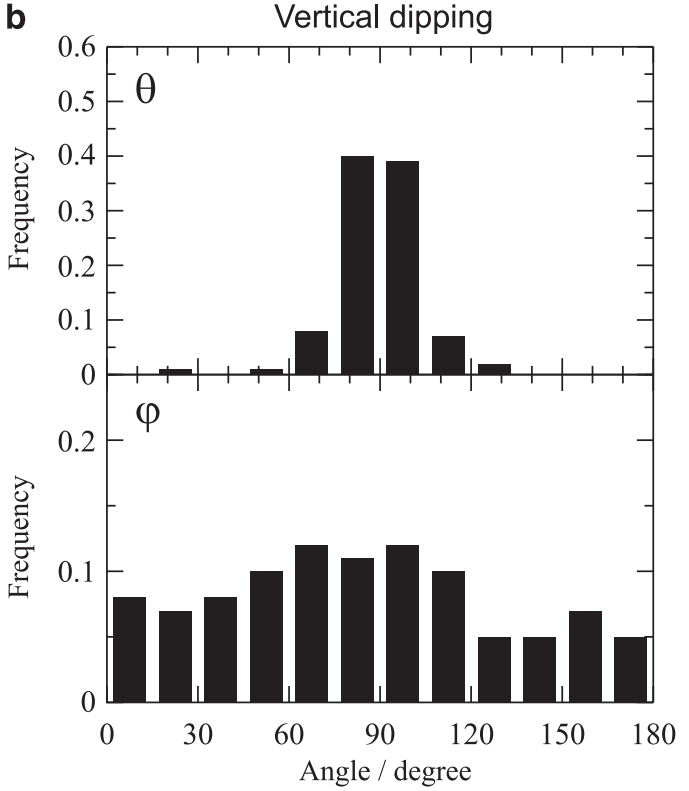

Figure 4 Histogram of the orientational angle for the monolayer deposited by the horizontal (a) and vertical dipping methods (b). The s.d. of the polar angle was 10.6 and 11.4 for the $\mathrm{H}$ - and V-films, respectively.

parallel to the main chain of PMMA. Therefore, the segmental orientation of the chain center directly corresponded to the direction of the PDI moiety, which could be evaluated in three dimensions with high precision as mentioned above. Thus, the orientation angle of the center segment of a single PMMA chain can be discussed using the defocused image. Figure 4 shows the histogram of the orientation of the PDI moiety in the PMMA monolayer. The distribution of the polar angle, $\theta$, showed a sharp peak at the angle of $90^{\circ}$ for both $\mathrm{H}$ - and V-films (the upper part in panels $\mathbf{a}$ and $\mathbf{b}$, respectively). In the case of three-dimensional random orientation for a spin-cast film, the distribution function of the polar angle $p(\theta)$ also showed the maximum population at $90^{\circ}$, with a broad dispersion in the range of $0^{\circ} \leqslant \theta<$ $180^{\circ}$, which is expressed as $p(\theta) \propto \sin \theta .^{15}$ For the monolayer sample, more than $80 \%$ of the PDI moiety at the center segment of the PMMA chain showed polar orientation in the angular range of $80^{\circ}<\theta<100^{\circ}$. The values of the s.d. of the polar angle for $\mathrm{V}$ - and $\mathrm{H}$-films were $11.4^{\circ}$ and $10.6^{\circ}$, respectively. These values were much smaller than those for randomly oriented films $\left(52.7^{\circ}\right)$. This clearly indicates that the center segment of the PMMA chain in a monolayer was aligned in the film plane.

Next, we discuss the azimuthal angle, $\varphi$. The H-film showed no peak in the histogram of $\varphi$ (the lower part of Figure 4a), indicating a random orientation of the azimuthal angle. However, the histogram for the V-film showed a weak peak at the angle of $90^{\circ}$, which corresponds to the dipping direction in the LB process. The statistical analysis of data for the individual molecules allows the discussion on the ensemble-averaged orientation. The average orientation in a given axis can be calculated by $\Phi=\left\langle\left\{(\mathbf{M} / M) \cdot \mathbf{e}_{0}\right\}^{2}\right\rangle$, where $\mathbf{e}_{0}$ is the unit vector in the considered direction, $\mathbf{M}$ is the transition dipole moment of each molecule and brackets \langle\rangle represent the average over all of the observed molecules. When all molecules are aligned in the direction of $\mathbf{e}_{0}, \Phi$ is unity. In the case of a random orientation in two- and three-dimensional systems, $\Phi_{\text {rand }}=1 / 2$ and $1 / 3$, respectively. The orientation function $f$ is defined as $f=\left(\Phi-\Phi_{\text {rand }}\right) /\left(\Phi_{0}-\Phi_{\text {rand }}\right)$, where $\Phi_{0}$ is the average orientation for the complete orientation along the direction of $\mathbf{e}_{0}$. For the spin-cast film, the maximum value of $f$ with respect to $\mathbf{e}_{0}$ in arbitrary directions was evaluated to be 0.045 from the single molecule detection, indicating a random orientation in three dimensions. On the other hand, the orientation function for the two-dimensional 
monolayer system was evaluated to be 0.0088 and 0.15 for the $\mathrm{H}$ - and V-films, respectively. For the H-film, this was the maximum value observed with respect to various directions of $\mathbf{e}_{0}$. For the V-film, $\mathbf{e}_{0}$ was parallel to the dipping direction. This indicates that the center segment of the PMMA chain in an LB film deposited by the vertical deposition process was weakly aligned in the dipping direction, whereas the horizontally deposited monolayer showed no preferential orientation in the film plane. The polymer chain on a water surface should show isotropic orientation of the segments because the segmental orientation was randomized because of high mobility on water. ${ }^{20}$ In the horizontal dipping method, the monolayer on water is transferred by touching the substrate plane to the subphase surface in parallel; consequently, the in-plane random orientation of the chain segment on water was maintained in the deposited monolayer. On the other hand, the immersed substrate was lifted from the subphase in the vertical deposition process, resulting in the flow of the monolayer. ${ }^{9}$ Consequently, the segment of the polymer chain in the monolayer was oriented by the flowinduced deformation. In the case of rigid chains such as DNA and polypetides in an $\alpha$-helix conformation, the polymer chain is strongly oriented in the dipping direction. ${ }^{9-12}$ However, the orientation of the PMMA main chain in a monolayer is weak compared with rigid chains. Because of the flexibility of the PMMA chain, the fast relaxation of the chain segment resulted in the weak orientation in the dipping direction.

\section{CONCLUSION}

The segmental orientation of a single polymer chain in a LB film of PMMA was examined at the molecular level. A fluorescence-labeled segment in the main chain was observed by fluorescence imaging in a defocused condition, which enabled the direct evaluation of the three-dimensional molecular orientation. For the azimuthal angle $\varphi$ in the film plane, the PMMA chain in an LB monolayer deposited by vertical dipping was weakly oriented in the direction of dipping. On the other hand, the polymer chain in the horizontally dipped film showed no preferential orientation. The polar angle showed a narrow orientational distribution of $\theta=90^{\circ}$, indicating that the main chain in the monolayer lies on a quasi-two-dimensional plane. This result indicates that the horizontally deposited monolayer is a good model to discuss the properties of the polymer chain in two dimensions.

\section{ACKNOWLEDGEMENTS}

This work was supported by Grants-in-Aid from the Ministry of Education, Culture, Sports, Science and Technology, Japan (MEXT). The authors also acknowledge the Innovative Techno-Hub for Integrated Medical Bio-imaging Project of the Special Coordination Funds for Promoting Science and Technology from MEXT.

\section{Hiroyuki Aoki ${ }^{1,2}$, Toshiaki Takahashi ${ }^{1}$ and} Shinzaburo Ito ${ }^{1,2}$

\section{${ }^{1}$ Department of Polymer Chemistry, Kyoto University, Nishikyo, Kyoto, Japan and ${ }^{2}$ Advanced Biomedical Engineering Research Unit, Kyoto University, Nishikyo, Kyoto, Japan \\ E-mail: aoki@photo.polym.kyoto-u.ac.jp}

1 Tredgold, R. H. \& Winter, C. S. Langmuir-Blodgett monolayers of preformed polymers. J. Phys. D Appl. Phys. 15, L55-L58 (1982).

2 Tredgold, R. H. Langmuir-Blodgett films made from preformed polymers. Thin Solid Films 152, 223-230 (1987).

3 Grohens, Y., Hamon, L., Reiter, G., Soldera, A. \& Holl, Y. Some relevant parameters affecting the glass transition of supported ultra-thin polymer films. Euro. Phys. J. E 8, 217-224 (2002).

4 Keddie, J. L., Jones, R. A. L. \& Cory, R. A. Sizedependent depression of the glass transition temperature in polymer films. Europhys. Lett. 27, 59-64 (1994).

$5 \mathrm{Si}$, L., Massa, M. V., Dalnoki-Veress, K., Brown, H. R. \& Jones, R. A. L. Chain entanglement in thin freestanding polymer films. Phys. Rev. Lett. 94, 127801 (2005).

6 Sato, N., Ito, S. \& Yamamoto, M. Molecular weight dependence of shear viscosity of a polymer monolayer: evidence for the lack of chain entanglement in the twodimensional plane. Macromolecules 31, 2673-2675 (1998).

7 Aoki, H., Anryu, M. \& Ito, S. Two-dimensional polymers investigated by scanning near-field optical microscopy: conformation of single polymer chain in monolayer. Polymer 46, 5896-5902 (2005).

8 Aoki, H., Morita, S., Sekine, R. \& Ito, S. Conformation of single poly(methyl methacrylate) chains in an ultrathin film studied by scanning near-field optical microscopy. Polym. J. 40, 274-280 (2008).

9 Schwiegk, S., Vahlenkampv, T., Xu, Y. Z. \& Wenger, G. Origin of orientation phenomena observed in layered Langmuir-Blodgett structures of hairy-rod polymers. Macromolecules 25, 2513-2525 (1992).

10 Okahata, Y., Kobayashi, T. \& Tanaka, K. Orientation of DNA double strands in a Langmuir-Blodgett film. Langmuir 12, 1326-1330 (1996).

11 Friedenberg, M. C., Fuller, G. G., Frank, C. W. \& Robertson, C. R. In situ optical studies of flow-induced orientation in a two-dimensional polymer solution. Macromolecules 29, 705-712 (1996).

12 Mabuchi, M., Kobata, S., Ito, S., Yamamoto, M., Schmidt, A. \& Knoll, W. Preparation and characterization of the Langmuir-Blodgett films made of hairly-rod polyglutamates bearing various chromophores in the side chain. Langmuir 14, 7260-7266 (1998).

13 Ito, S., Kanno, K., Ohmori, S., Onogi, Y. \& Yamamoto, M. Fluorescence polarization method for studying molecular-orientation of monolayered and multilayered polyimide films prepared by the LangmuirBlodgett technique. Macromolecules 24, 659-665 (1991).

14 Patra, D., Gregor, I. \& Enderlein, J. Image analysis of defocused single-molecule images for three-dimensional molecule orientation studies. J. Phys. Chem. A 108, 6836-6841 (2004).

15 Aoki, H., Takahashi, T., Tamai, Y., Sekine, R., Aoki, S., Tani, K. \& Ito, S. Poly(methacrylate)s labeled by perylene diimide: synthesis and applications in single chain detection studies. Polym. J. 41, 778-783 (2009).

16 Dedecker, P., Muls, B., Deres, A., Uji-i, H., Hotta, J., Sliwa, M., Soumillion, J., Müllen, K., Enderlein, J. \& Hofkens, J. Defocused wide-field imaging unravels structural and temporal heterogeneity in complex systems. Adv. Mater. 21, 1079-1090 (2009).

17 Livanec, P. W. \& Dunn, R. C. Single-molecule probes of lipid membrane structure. Langmuir 24, 14066-14073 (2008).

18 Nishimura, D., Takashima, Y., Aoki, H., Takahashi, T., Yamaguchi, H., Ito, S. \& Harada, A. Single-molecule imaging of rotaxanes immobilized on glass substrates: observation of rotary movement. Angew. Chem. Int. Ed. 120, 6166-6168 (2008).

19 Toprak, E., Enderlein, J., Syed, S., McKinney, S. A., Petschek, R. G., Ha, T., Goldman, Y. E. \& Selvin, P. R. Defocused orientation and position imaging (DOPI) of myosin v. Proc. Natl Acad. Sci. USA 103, 6495-6499 (2006).

20 Sato, N., Sugiura, K., Ito, S. \& Yamamoto, M. Molecular motion in polymer monolayers at the air/ water interface. A time-resolved study of fluorescence depolarization. Langmuir 13, 5685-5690 (1997).

Supplementary Information accompanies the paper on Polymer Journal website (http://www.nature.com/pj) 